\title{
An overall comparison of small molecules and large biologics in ADME testing
}

\author{
Hong Wan \\ Shanghai Hengrui Pharmaceutical Co., LTD. Dept. of DMPK/Tox, Shanghai, P.R. China \\ E-mail: wanh@shhrp.com; Tel.: +86(0)21-54759066-1313
}

Received: March 12, 2016; Revised: March 23; Published: March 31, 2016

\begin{abstract}
Biologics mainly monoclonal antibodies (mAbs) and antibody-drug conjugates (ADCs) as new therapeutics are becoming increasingly important biotherapeutics. This review is intended to provide an overall comparison between small molecules (SMs) and biologics or large molecules (LMs) concerning drug metabolism and pharmacokinetic (DMPK) or associated with absorption, distribution, metabolism and elimination (ADME) testing from pharmaceutical industry drug discovery and development points of view, which will help design and conduct relevant ADME testing for biologics such as mAbs and ADCs. Recent advancements in the ADME for testing biologics and related bioanalytical methods are discussed with an emphasis on $A D C$ drug development as an example to understand its complexity and challenges from extensive in vitro characterization to in vivo animal PK studies. General non-clinical safety evaluations of biologics in particular for $A D C$ drugs are outlined including drug-drug interaction (DDI) and metabolite/catabolite assessments. Regulatory guidance on the ADME testing and safety evaluations including immunogenicity as well as bioanalytical considerations are addressed for LMs. In addition, the preclinical and human PK data of two marked ADC drugs (ADCETRIS, SGN-35 and KADCYLA, T-DM1) as examples are briefly discussed with regard to PK considerations and PK/PD perspectives.
\end{abstract}

\section{Keywords}

monoclonal antibody; antibody-drug conjugate; pharmacokinetics; bioanalysis; preclinical safety

\section{Introduction}

Biologics or large molecules (LMs) primarily monoclonal antibodies (mAbs) and antibody-drug conjugate (ADC) currently represent main stream therapeutics and continue to grow in number of new approvals and targets recently [1-4]. A majority of these biotherapeutics are mAbs or mAb-derived proteins, which are subject to transformation mechanisms such as deamidation, oxidation, and isomerization [1-4]. These processes usually result in relatively small structural changes in the parent drugs. Such small structural changes may be difficult for a conventional immunoassay to differentiate, but they can still affect biological activity, PK and immunogenicity of a therapeutic protein [5]. Whereas ADCs are an emerging class of biotherapeutics that combines the target specificity of an antibody with the potent small-molecule drugs or cytotoxins, which can selectively deliver a potent cytotoxic drug to tumor cells via tumor-specific and/or over-expressed antigens with more favorable therapeutic window [1,6]. This new type of antibody-drug conjugate or antibody-linker-drug currently shows its great promising therapeutic options, which led to the recent FDA approvals of ADCETRIS (brentuximab vedotin, SGN-35) and KADCYLA (ado-trastuzumab 
emtansine, T-DM1) for the treatment of Hodgkin's lymphoma (HL) and anaplastic large-cell lymphomas (ALCL), and HER2-positive metastatic breast cancer, respectively, as well as a rich clinical pipeline of potential new cancer therapies [7].

Throughout several decades of advancements and evolutions, ADMET profiling of small molecule (SM) has becomes a standardized paradigm in drug discovery and development in terms of in vitro screening, in vivo animal studies, LC-MS/MS based bioanalysis as well as regulatory considerations including DDI and drug metabolite safety testing etc. However, ADME testing of LMs lags behind that of SMs due to the complex nature of the biological molecules and also lack of appropriate tools to study drug exposure, biotransformation and target engagement in the vascular and tissue spaces [2]. ADME of LMs or biologics are still based on the lessons learned from the SMs and the tools that have applied to SM drugs [5]. In general, there is a similar high level PK/PD relation concept between the SMs and LMs, although they have different ADME mechanisms and underlying ADMET determinants at different stages [5]. Characterizing the absorption, distribution, metabolism, and excretion of these LM drugs (mAbs and ADCs) in preclinical animal models can better predict their efficacy and tolerability in clinic. Accordingly, it is necessary to understand general characteristics and the difference between SMs and LMs (e.g., antibody and ADC) in order to apply relevant approaches for ADME testing and safety evaluation of LMs as reviewed in a number of recent publications $[2,3,5,8-11]$. In a previous review, what ADME tests should be conducted for small molecule drugs for preclinical studies was highlighted [12]. This review will provide an overall comparison between SM and LM properties with a particular focus on ADC's characteristics to gain a better understanding of in vitro and in vivo ADME testing as well as toxicity evaluations. The in-depth information of ADME will also be valuable for the designing of novel mAb constructs and next generation of ADCs with desirable PK profile and safety window.

\section{General differences between $S M s$ and $L M s$ (mAb and ADC) in ADME testing}

Table 1 summarizes general differences between SMs and LMs with an overall comparison of mAb and ADC. As highlighted in Table 1, due to the nature of various characteristics between SMs and LMs, the focus on ADME studies of LMs is thus different from SMs in particular for drug metabolite safety testing and DDI evaluations. In these aspects, in vitro models and in vivo studies and related bioanalysis including transporter studies and safety evaluation and high-throughput screening approaches for SMs have well been established across pharmaceutical industry [12-20]. Typical in vitro ADME tests for SMs are metabolic stability by liver microsmoes or/and hepatocytes and passive permeability on cell-line models based on Caco-2 or MDCK assays, which are commonly utilized to predict in vivo clearance and absorption or bioavailability as well as potential drug-drug interaction (DDI) evaluations and metabolism pathway studies. In particular, a common consensus has been reached on drug metabolite testing across various species from in vitro models to in vivo studies following regulatory guidance, which clearly suggests critical criteria for decision-tree making in assessing key drug metabolite safety in human [21,22]. Similarly, cytochrome P450 (CYP) enzymes based DDI as well as transporter-mediated DDI of SMs are also defined and guided on the basis of many years industrious practice of drug discovery and development [23]. On the contrary, ADME testing of biologics ( $m A b s$ and ADCs) can be rather diverse as highlighted in Table 1 and Figure 1. Biodistribution of mAbs and ADCs are usually similar [24], but both have much lower $V_{d}$ than that of a typical SM drug as the structure of mAbs or ADCs is dominated by the antibody backbone with initial distribution limited to the vascular space or plasma, not organ tissues. While the drug metabolism of mAbs is more complex due to receptor binding target-mediated drug disposition (TMDD), FcRn binding and Iysosomal degradation, tissue protease, immune response antibody mediated metabolism etc in addition to 
other metabolic or metabolic-like biotransformation such modifications as deamidation, oxidation, isomerization, disulfide bond reduction or shuffling and proteolytic or glycolytic hydrolysis (ref. therein) [11]. The metabolism /catabolism of ADCs can be more complicated than mAbs due to a cytotoxic drug linked to the antibody via a linker. Typically, the circulating unconjugated drug after the ADC administration has metabolic properties of small-molecule compounds. Hence, drug metabolite and DDI should be concerned for ADC drugs, but maybe not for mAbs wherein the DDI risk is presumably low or not as prominent as small molecules. Once small-molecule drugs released from the ADCs, they may be metabolized by CYP enzymes and thus subject to potential DDI from CYP enzyme inhibitors or inducers due to payload/small molecule component [9]. Furthermore, one or more active small-molecule drugs may be released from an ADC in vivo by additional catabolism mechanism. Accordingly, both unconjugated smallmolecule drug and released catabolites small molecules may be considered for metabolite safety and DDI potential evaluations for ADCs. This is considered to be a major differentiation of ADCs from mAbs regarding ADME testing as the degraded small molecules of mAbs are often amino acids, small peptides or small carbohydrates that are readily eliminated by renal excretion or return to the nutrient pool without biological effects or safety consideration [2]. However, it should be noted that examples of therapeutic protein (TP) and small-molecule drug (D) interactions in clinical studies were observed although the changes in exposures (AUC, Cmax) have not been as remarkable as with small-molecule drugs, and the types of study designs used to assess TP and D interactions were thereby outlined in CDER's special subject on therapeutic protein-drug interactions and implications for drug development [25].

On the other hand, for LMs at early stage, more extensive in vitro characterizations are required as an example of screening cascade depicted in Figure 1. These typical studies include antibody primary structure by chemical sequencing (Edman degradation) as well as peptide mapping by means of ESI-MS and MALDIMS [26-28]; higher order structure by RP-HPLC-ESI-MS, Ellman's assay CD, FTIR, hydrogen deuterium exchange (HDX)-MS and X-ray [28,29]; post-translational modifications (PTM) and charge variants by MSion-exchange chromatography (IEC), hydrophobic interaction chromatography (HIC), capillary electrophoresis (CE) and isoelectric focusing (IEF) [30]; glycan profile and variants and size heterogeneity by HPLC-fluorescence, size-exclusion chromatography (SEC), native gel, capillary electrophoresis, RP-HPLC-MS and native intact MS [31], as well as solubility measurement by ultrafiltration or PEG-induced precipitation methods [32,33]. Moreover, the immunogenicity should be evaluated for both antibody and ADCs [34,35], but unnecessary for SMs. Unlike the antibody, additional in vitro studies of ADC include drug antibody ratio (DAR) characterizations, conjugate site analysis, linker stability and toxin evaluations as highlighted in Figure 1 may be required. Furthermore, absorption needs to be understood as well if LMs are targeting for an SC administration.

Despite distinct characteristics and differences of in vitro testing between antibody and ADCs and SMs as shown in Table 1 and Figure 1, similar principle of in vivo PK studies including mass balance and elimination as well as safety evaluations for SMs are generally applicable to both antibody and ADCs. However, different bioanalytical methods have to be applied for antibody and ADCs, which will be discussed in more details in bioanalytical section. 
Table 1. Characteristic comparison of small molecules (SMs) and large molecules (LMs)*

\begin{tabular}{|c|c|c|c|}
\hline & \multirow[t]{2}{*}{ SMs } & \multicolumn{2}{|c|}{ Biologics or LMs } \\
\hline & & mAbs & ADC \\
\hline MW & $\sim 200-500$ & 〜150 KDa (typically) & $\sim 150$ KDa (typically) \\
\hline Test article & Chemical & Protein & Conjugate (protein-chemical) \\
\hline $\begin{array}{l}\text { Physicochemical } \\
\text { properties }\end{array}$ & $\begin{array}{l}\text { Mostly well-defined } \\
\text { physicochemical properties }\end{array}$ & $\begin{array}{c}\text { Complex physicochemical } \\
\text { properties (e.g. tertiary structure, } \\
\text { stability, PTM) }\end{array}$ & Antibody + toxin \\
\hline ADME tools & $\begin{array}{c}\text { Available/ extensive ADME } \\
\text { understanding }\end{array}$ & $\begin{array}{c}\text { Understanding of ADME still } \\
\text { evolving }\end{array}$ & Understanding of ADME still evolving \\
\hline Dosing route & Oral often possible & $\begin{array}{l}\text { Usually parenterally (IV, SC, and } \\
\text { IM), Intravitreal injection }\end{array}$ & Usually parenterally (IV, SC, and IM) \\
\hline Dose interval & Daily (typically) & Intermittent dosing & Intermittent dosing \\
\hline Half-life $\left(t_{1 / 2}\right)$ & Short (typically several to $24 \mathrm{hrs}$ ) & Long (typically days or weeks) & $\begin{array}{l}\text { Long but slightly shorter than antibody } \\
\text { alone }\end{array}$ \\
\hline Distribution $\left(V_{d}\right)$ & $\begin{array}{c}\text { High } V_{\mathrm{d}} \text {, distribution to } \\
\text { organs/tissues }\end{array}$ & $\begin{array}{l}\text { Lower } V_{d}, \text { usually limited to } \\
\text { plasma and/ or extracellular } \\
\text { fluids }\end{array}$ & Lower $V_{d}$, mainly target dependent \\
\hline $\begin{array}{l}\text { Metabolism } \\
\text { pathway }\end{array}$ & $\begin{array}{l}\text { Mainly by CYP enzymes and phase } \\
\text { II enzymes, metabolized to non- } \\
\text { active and active metabolites }\end{array}$ & $\begin{array}{c}\text { Catabolism } \\
\text { Degraded to peptides or amino } \\
\text { acids }\end{array}$ & Both catabolism and metabolism \\
\hline $\begin{array}{l}\text { Drug metabolite } \\
\text { safety evaluation }\end{array}$ & Yes & No & $\begin{array}{l}\text { Toxin and released drug catabolites } \\
\text { (may be concerned) }\end{array}$ \\
\hline Excretion & Mainly biliary and renal excretion & Mostly recycled by body & Both \\
\hline Clearance (CL) & $\begin{array}{c}\text { Mostly linear PK; non-linearity } \\
\text { mainly due to saturation of } \\
\text { metabolic pathways }\end{array}$ & Slow clearance & $\begin{array}{l}\text { Lower clearance, but slightly higher } \\
\text { clearance than antibody }\end{array}$ \\
\hline $\begin{array}{l}\text { Potency and } \\
\text { selectivity }\end{array}$ & Generally less selective & High selectivity (affinity/ potency) & High selectivity (affinity/ potency) \\
\hline PK analytes & Drug and metabolites & Antibody and ADA & $\begin{array}{c}\text { ADC or conjugated drug, total antibody, } \\
\text { and unconjugated toxin/catabolites, } \\
\text { ATA }\end{array}$ \\
\hline PK bioanalysis & LC-MS/MS methods & $\begin{array}{l}\text { Mostly ELISA (total antibody), } \\
\text { Recently with increased LC- } \\
\text { MS/MS applications }\end{array}$ & $\begin{array}{l}\text { Hybrid of ELISA (total antibody, ADC), } \\
\text { high resolution LC-MS (DAR) and highly } \\
\text { selective LC-/MS/MS (un-conjugated } \\
\text { toxin/catabolites) }\end{array}$ \\
\hline PD & Short acting & Long acting & Long acting \\
\hline PK/PD & $\begin{array}{l}\text { PK usually not driven by PD due to } \\
\text { dominance of non-target mediated } \\
\text { binding }\end{array}$ & $\begin{array}{l}\text { PK and PD mechanistically } \\
\text { connected (TMDD) }\end{array}$ & $\begin{array}{l}\text { PK and PD mechanistically connected } \\
\text { (TMDD) }\end{array}$ \\
\hline DDI & $\begin{array}{l}\text { Many examples and PK and/or PD } \\
\text { related (by CYP enzymes or } \\
\text { transporters) }\end{array}$ & $\begin{array}{l}\text { Sparse examples and mostly PD } \\
\text { related }\end{array}$ & $\begin{array}{c}\text { Sparse examples and mostly PD related } \\
\text { Toxin or released toxin/catabolites } \\
\text { (should be concerned) }\end{array}$ \\
\hline hERG & Yes & No & No \\
\hline Immunogenicity & No & Yes & Yes \\
\hline Toxicity & On-and off-target related toxicity & $\begin{array}{l}\text { Typically exaggerated } \\
\text { pharmacology }\end{array}$ & $\begin{array}{c}\text { Typically antigen-independent } \\
\text { Can be more toxic than antibody due to } \\
\text { toxin }\end{array}$ \\
\hline Formulation & Complex and diverse & Simple formulation & Simple formulation \\
\hline $\begin{array}{l}\text { API/Production } \\
\text { Process }\end{array}$ & $\begin{array}{l}\text { Synthesized ( uniform single entity) } \\
\text { Generics, bioequivalence }\end{array}$ & $\begin{array}{l}\text { Culture-derived (generally } \\
\text { nonuniform) } \\
\text { No generics, biosimilar or } \\
\text { comparability }\end{array}$ & $\begin{array}{l}\text { Both + conjugation (mixtures of ADCs } \\
\text { with different DAR } \\
\text { No generics, biosimilar or comparability }\end{array}$ \\
\hline
\end{tabular}

\footnotetext{
*Modified from references [8-10].
} 


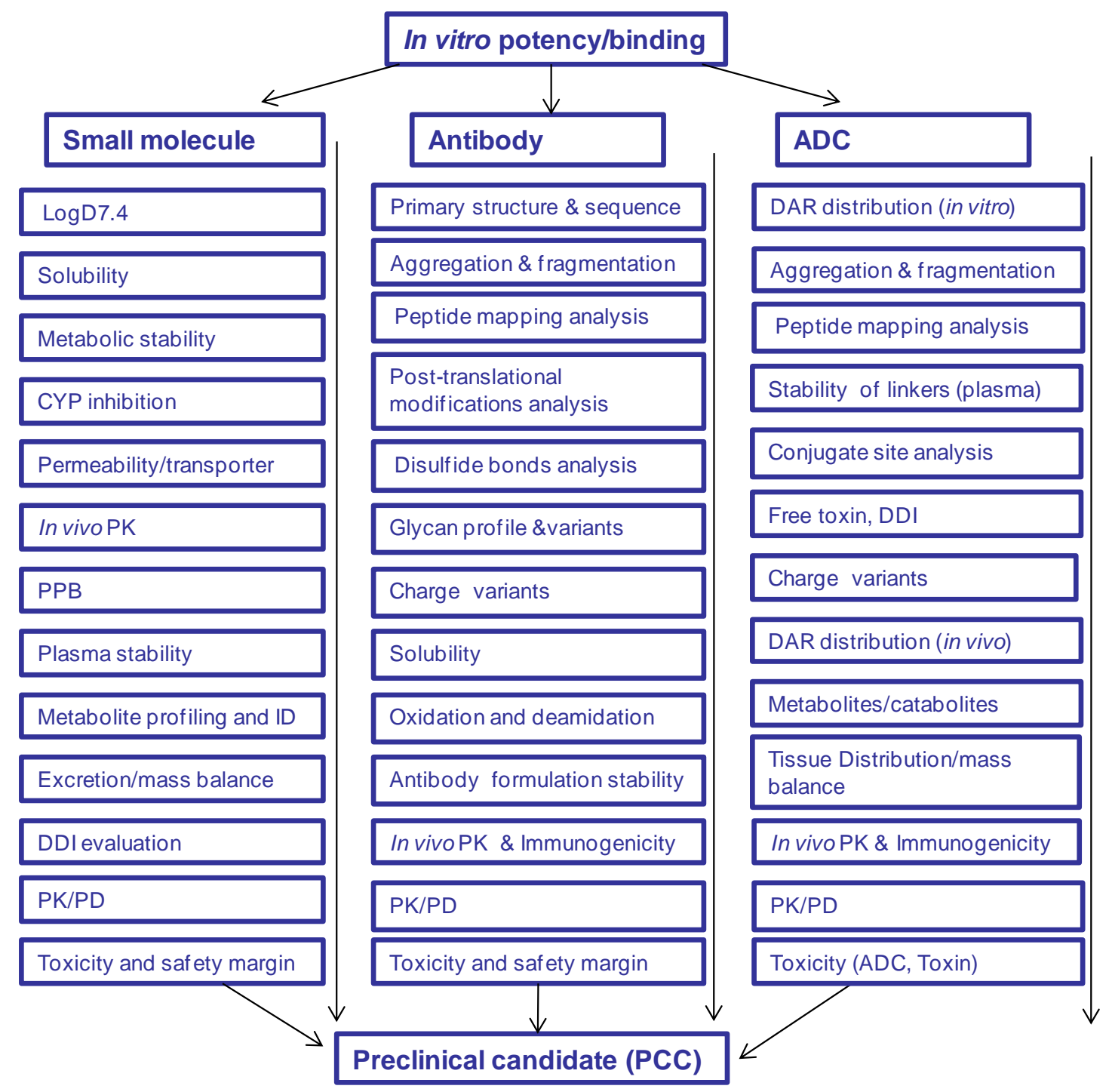

Figure 1. Typical ADME/Tox studies for SM and antibody and ADC

\subsection{ADME testing of $m A$ bs}

Fundamental ADME characteristics and PK behaviors of biologics including therapeutic proteins and mAbs have extensively been reviewed recently [2,11,25,36-39]. Currently, there is lack of validated in vitro ADME models for mAbs. In addition to extensive characterization of antibody as depicted in Figure 1, the major ADME testing of mAbs relies on in vivo studies such as PK and safety evaluations on relevant animal species. It should be noted that the selected species for in vivo PK and toxicity evaluations should preferably have cross-reactivity with human. Another important aspect to consider is immunogenicity for ADME testing of mAbs as well as ADCs, which is a key risk in biologic drug development, because therapeutic biotechnology-driven proteins must be as similar as possible in primary, secondary, and tertiary structures to its human homologues in order to avoid immunogenicity [2,40]. In general, the immunogenicity, specifically formation of anti-drug antibody (ADA), is one of the major complicating issues in nonclinical and clinical programs for therapeutic proteins, which is a regulatory requirement as part of the safety assessment of biotherapeutic submission [34,38,41]. The ADA production due to the continuous administration of mAbs may affect the PK and/or the PD of mAbs after they are administrated to patients [40]. When an immunogenic reaction mediated by ADAs takes place, it can increase blood clearance and decreases exposure (often relatively shorter half-life observed), thereby reducing its efficacy and likely 
hampering its clinical application in some instances. Although factors that affect ADA are complex and the relationship of bioanalytical measures, for example, incidence and magnitude of ADA responses to clinical efficacy and safety are uncertain, the ADA incidence and magnitude should always be assessed relative to capacity of ADAs to neutralize the relevant biological activity of the therapeutic mAb, in addition to impact on drug levels and clinical measures of efficacy and safety [42]. In principle, it would be acceptable for the biologic product to be less immunogenic than the reference product, provided that this did not modify the efficacy of the product or increase the incidence or severity of adverse reactions, although the magnitude of ADA acceptance is not defined by regulators [42]. One should also bear in mind that the bioanalytical methods used to monitor immunogenicity are subject to various biases when interpreting ADA data and assessing immunogenicity. In practice, a comparison of single dosing PK and multiple dosing PK with monitoring ADA production over time is valuable for a better understanding of the PK/PD relation of mAbs as well as the part of mAb quality evaluation. To the best of our knowledge, once the ADA occurs over the time, the plasma concentration at certain time point can drop dramatically. Consequently, caution has to be taken to evaluate the PK parameters in particular calculate the half-life of mAb with different compartmental models to be in line with its corresponding plasma concentrations for the correct interpretation of PD effect or its PK/PD relationship. Additionally, mathematical modeling of ADA response against mAbs and its impact on mAb PK/PD has been reviewed recently [40].

Currently, ligand binding assay (LBA) such as enzyme-linked immunesorbent assays (ELISA) are generally regarded as the gold standard methods for bioanalysis of LMs with sufficient sensitivity and specificity. Of them, the sandwich and competition enzyme immunoassay technique are more widely utilized for detection and quantification of antibodies [43-45]. Typically, various coat capture reagents, conjugate reagents (e.g. horseradish peroxidase, HRP), peroxidase substrates (e.g. TMB, stabilized hydrogen peroxide/TMB) and stop solution (e.g. sulfuric acid) are employed case by case. To date, the standard ELISA platform has considerably been improved upon over the years, involving automation and improved detection methodologies, and new applications of this technique is further discussed in next bioanalysis section.

\subsection{ADME testing of $A D C s$}

$A D C s$ are mAbs with covalently bound cytotoxic drugs via a linker, which are designed to target tumor antigens selectively and offer the hope of cancer treatment to decrease the off-target toxicity, thereby, improving the therapeutic index of the cytotoxin $[41,46]$. ADCs are usually comprised of 0 to 8 cytotoxic payloads with an average DAR of 2 to 4 per mAb, which are thus heterogeneous mixtures of conjugates. Given that low payloads reduce potency and high payloads negatively impact PK, the DARs can have a significant impact on ADC efficacy [3]. Structurally, the antibody component of the ADC accounts for the majority of the therapeutic agent (approximately $98 \%$ of total ADC by molecular weight). Biologically, the PK of ADCs is strongly influenced by the underlying antibody backbone conferring properties such as target specific binding, neonatal Fc receptor ( $F C R n)$-dependent recycling and Fc (fragment, crystallizable) effector functions [24]. Compared with the unconjugated antibody, ADCs can exhibit somewhat higher clearance due to introduction of an additional metabolic pathway (i.e. cleavage of the drug from the antibody by lysosomal peptide degradation). Furthermore, linker stability, DAR and site of drug conjugation can largely impact PK behaviors and distribution as well. Conjugation with higher DAR tends to have faster clearance than conjugation with lower DAR as an example of cAC10-vc MMAE ADCs with high DARs exhibiting a faster total antibody clearance than lower DAR ADCs [47]. Variable DARs and attachment sites by a consequence of current random conjugation methods result in heterogeneous ADCs with PK parameters that can vary substantially compared to the unconjugated antibody [24]. Hamblett et al. compared ADCs with different 
drug loading (MMAE conjugated to the anti-CD30 mAb) and concluded that the therapeutic index was increased by decreasing drug loading per antibody, demonstrating that drug loading is a key design parameter for antibody-drug conjugates [47]. It was shown that the terminal half-lives did not directly correlate with drug loading. When the drug loading increased, both clearance and volume of distribution increased, but the exposure (AUC) of ADC decreased, reflecting the significant attribute of DAR to PK properties of ADC.

The systemic stability of the antibody-drug linker is also crucial for delivery of an intact ADC to targetexpressing tumors. Linkers stable in circulation but readily processed in the target cell are necessary for both safety and potency of the delivered conjugate [48]. In general, linker should be stable enough in systemic circulation to deliver drug into tumor cells, but labile to release this fully active payload drug inside the tumor cells. Otherwise, the toxic drug could be released in the bloodstream, causing systemic toxicity. Polson et al. demonstrated that non-cleavable linker (e.g. SGN-35) is more stable than cleavable linker (e.g. T-DM1) [49]. The non-cleavable linker may have slower deconjugation or lower clearance than cleavable linker and reduced systemic toxicity of $A D C$ in rats presumably due to the reduced release of free drug or other toxic metabolites into the circulation [49]. This is because the cleavable linkers may release drug by lysosomal proteases without the degradation of the mAb component whereas non-cleavable linkers require catabolism of the mAb backbones to release the drug [9 and ref. therein]. In addition, the selection of attachment site can affect stability of cleavable antibody-drug conjugates, as demonstrated in the design of systemically stable cleavable auristatin-based conjugates, offering the means to overcome inherent linker instability by optimal attachment site [48].

Interestingly, Junutula et al. [50] recently reported a site-specific conjugation method called THIOMAB technology enabling near-uniform, low-level conjugation of cytotoxins ( $D A R=2)$ to antitumor antibodies, which displayed an increased tolerability or improved therapeutic index without compromising efficacy. This new type of THIOMAB-drug conjugates (TDCS) with nearly homogeneous composition of conjugates was better tolerated than conventional $A D C$ in monkeys, and also showed slower catabolism and deconjugation than ADC in rats. The THIOMAB approach provides a general strategy for improved PK and superior therapeutic index of any ADC generated by standard sufhydryl-directed maleimide chemistry [51]. Likewise, Dennler et al. more recently presented another site-specific modification of deglycosylated antibodies by microbial transglutaminase (MTGase) to form ADCs with a defined homogeneous DAR of 2 as well. This chemo-enzymatic approach was reported directly applicable to abroad variety of antibodies as it does not require prior genetic modifications of the antibody sequence [52]. Clearly, the designing and forming a homogeneous ADC can result in less complicated in vitro characterization and relatively easier in vivo PK bioanalysis as well as safety evaluation than a typical heterogeneous ADC.

Apart from the DAR characterization, some in vitro ADME models and in vivo PK studies can be employed for $A D C$ evaluations as illustrated in Figure 1. First ADME testing of ADCs is linker stability assessment both in in vitro or in vivo by LC-MS/MS method or radiolabeled ADC, respectively, in order to explore the impact of linker on uptake and catabolism. Secondly, free toxin was expected to undergo clearance mechanism studies for DDI potential including liver microsomal stability, metabolizing enzyme identification, CYP inhibition and induction, in vitro metabolite profiling studies [53]. Then, general in vivo PK studies and non-clinical safety evaluations including immunogenicity with relevant animal species are necessary for PCC selection. Finally, linker-containing drug catabolites should be identified and the plasma concentration of free drug and related catabolites should be determined by LC-MS/MS in both nonclinical and clinical studies for safety assessment of key metabolites or catabolites. 
ELISA was conventional method for PK/TK (toxicokinetics) study of antibody and conjugate. For instance, detection reagents for total and conjugated antibodies were goat antihuman IgG antibody conjugated to horseradish peroxidase or goat antihuman Fc conjugated to horseradish peroxidase [43]. Coat capture reagents were used on a case by case basis, e.g. anti-MMAE antibody (conjugated antibody), antibodies specific for drugs (total antibody). Biotin-DIG bridging ELISA and surface plasma resonance (SPR) techniques were developed for immunogenicity assessment to detect anti-therapeutic antibodies (ATAs) to the ADC, including ATAs towards any of the ADC molecular components such as the antibody, linker, drug or epitopes involving multiple ADC components. Finally, isotope-labelling method was used for the study of tissue distribution and mass balance $[43,46,54]$. Recent advances in high-throughput formats and combined techniques for ADC bioanalysis will be discussed in more details in the section 3 .

\subsection{Safety evaluation of $A D C S$}

With regard to preclinical safety assessment, toxicity of ADCs is usually $A D C /$ drug-dependent and antigen-independent and it can be more toxic than mAbs due to introduced cytotoxic drugs. As ADCs consist of a monoclonal antibody, linker and cytotoxic components, the biological activity profiles of each should be considered when selecting the relevant and/or appropriate species for toxicity evaluations, typically in two relevant species (one rodent and one non-rodent) [55]. For a particular ADC, the relevant toxicity species should be selected from its pharmacology and tissue-reactivity studies showing similar binding to human. Primary considerations for the nonclinical safety assessment of ADCs includes the evaluation of the entire ADC or the various individual components (i.e., antibody, linker or the cytotoxin) to identify the on- and off-target toxicities to enable first-in-human (FIH) studies [41]. General toxicity studies are recommended and following specific toxicity studies may be considered as well for overall nonclinical safety evaluations $[25,35,41,42,56,57]$ :

- Acute and chronic toxicity study on two relevant species (preferably rodent and non-rodent), or transgenic animals (i.e., animal modified to the human target) to attain maximum tolerated dose (MTD), non-observed adverse effect level (NOAEL) or the highest non-severely toxic dose (HNSTD).

- Tissue cross-reactivity (TCR) in tissue panels from humans and toxicity species for ADC and unconjugated antibody

- Genotoxicity of cytotoxin and/or linker, and ADC may be concerned

- hERG for unconjugated drug if concentration of the unconjugated cytotoxin are detected in the serum during toxicity and plasma stability testing

- Reproductive and developmental toxicity for embryofetal (EFD) toxicity assessment of ADC and unconjugated toxin

- Carcinogenicity

- Immunogenicity and anti-ADC antibody

- CYP inhibition and induction for DDI assessment

- Hemolysis, skin irritation and sensitization testing for biologics via injection administration.

Another important consideration would be the concentrations of unreacted linker that remain in the final drug product. If the cytotoxin is a novel chemical entity, then a more detailed assessment of metabolism and disposition (including PK/ADME studies) may be necessary as the cytotoxic drug released from the ADC may be associated with loss of efficacy or increased toxicity. However, if the concentrations of unconjugated cytotoxin in plasma are very low, for instance, the free DM1 after administrating the Trastuzumab emtansine (T-DM1) was $<10 \mathrm{ng} / \mathrm{ml}(<10 \mathrm{nM})$ at all doses [53], or maximal DM1 concentration did not exceed $25 \mathrm{ng} / \mathrm{ml}$ after repeated dosing of T-DM1 [58], the investigation of inhibition or induction of 
CYP isoenzymes to assess the potential DDI should not be necessary in this case [41]. In other words, the risk of $D D I$ is presumably low due to very low concentrations of cytotoxic drug released from ADCs. Despite this hypothesis, CYP3A4 based inhibition studies for KADCYLA and DECETRIS have been conducted, respectively, as the SGN-35 is metabolized with a small fraction of MMAE via oxidation by primarily CYP3A4 and CYP3A5, and KADCYLA (T-DM1) mainly by CYP3A4 and a lesser extent by CYP3A5. Nevertheless, an in vitro stability of $A D C s$ in plasma and in vivo monitoring toxin released from $A D C$ should be conducted to ensure the cytotoxic drug or potential catabolites under the minimal level as such without safety issues, in particular for major CYP metabolism involved ADC drugs.

On the other hand, a typical ADC consists of a distribution of several entities containing different numbers of cytotoxic drugs on antibody, linked at different positions, thus, the inherent heterogeneity of ADC complex with multiple components still remains a prominent challenge in understanding their properties in vivo during nonclinical development. For instance, conjugation through interchain disulfides can lead to these ADCs with DAR distribution ranging from 0 to 8 , with each fraction potentially exhibiting a unique efficacy, PK property, and toxicity profile [43]. Therefore, development of more sensitive and specific bioanalytical methods to differentiate a single ADC and multiple ADCs would be highly valuable to enable quantifying $A D C$ complex for a better understanding of attributes of each single ADC component to PK and PD as well as safety profiles. Although it would be ideal to monitor each individual ADC species of a specific DAR, this currently seems to be a technical challenge. Therefore, the most common approach is to monitor all ADC species, all antibody species or total antibody (TAb), and the unconjugated small molecule. An alternative approach is to monitor conjugated small molecules as a surrogate for ADC [9].

Overall, a design goal of an ADC is to maximize delivery of the cell-killing agent to the tumor tissue while minimizing delivery to normal tissues [54]. The choices of mAbs, linker, toxin or cytotoxic drug are all important determinants of PK, efficacy and safety. As a result, design and conduct of relevant ADME testing and fronting loading PK/PD studies as well as the key safety evaluations of lead molecules as early as possible will facilitate PCC's selection for their successful development.

\section{Bioanalytical methods and challenges}

In the past several decades of development of SMs, the bioanalytical strategies and approaches of SMs have been well established from method development, validation as well as method transfer from instrument to instrument and lab to lab based on LC-MS/MS according to FDA bioanaltical method guidance [59]. Differently from SMs, the most commonly used bioanalytical methods for LMs are ELISA based assays by either direct or bridging ELISA measuring the concentration of the therapeutic over time in plasma or serum for in vivo studies case by case. In comprehensive reviews and white papers, several bioanalytical methods, considerations and strategies as well as challenges have extensively been discussed for LMs [42,46,60-66]. This section will provide an overview on recent advancements in bioanalysis of mAbs, ADC, in particular with ELISA and LC-MS/MS technologies and related new approaches dealing with matrix interference.

\subsection{High-throughput bioanalysis}

From industry drug screening perspective, commercially automated Meso Scale Discovery (MSD) format and nonoscale immunoassay platform on Gyrolab offer high-throughput capabilities of ELISA bioanalysis, which enhances overall performance of traditional manual ELISA assay with several advantages in terms of sample amount, broader dynamic linearity, throughput, higher sensitivity and reproducibility $[67,68]$. Moreover, both the antibody and ADA of an antibody or ADC and ATA from PK plasma samples can be 
quantified simultaneously. In particular, the nanoscale volume of microfludic platform on Gyros has made it durable to gain the PK data of antibody from one single mouse study by whole blood serial sampling with significantly reducing study cost and animal usage, offering comparable PK data as conventional composite sampling [69]. Furthermore, this microfludic approach has been applied to high-throughput quantification of host cell protein impurities for bioprocess development [70]. It demonstrated an improved throughput (5-10 times faster), broader dynamic range (100-times) and decreased sample consumption, hands on time and duration for assay development compared with Tecan plate-based ELISA [70]. With those automated instruments, ELISA based bioanalytical methods become easier transferring from preclinical to clinical between labs and CROs, which can considerably speed up large molecule drug discovery process and clinical development. Furthermore, traditional ELISA approach was transferred to an automated microfluidics immunoassay platform based on nanoscale streptavidin bead columns enable highthroughput bioanalysis of a human mAb in preclinical PK samples with enhanced bioanalysis capacity [71]. Additionally, a high-throughput capillary electrophoresis based microfluidic device (LabChip GXII) was applied to obtain pharmacokinetics (PK) of a fluorescently labeled human mAb directly in single dose rat PK studies [72].

\subsection{LC-MS/MS and BLA bioanalysis of $m A$ Abs}

As mentioned above, LBA-based ELISA is the commonly used gold standard method for bioanalysis of mAbs. Recent advances in instrumentation technology have significantly increased the sensitivity and versatility of LC-MS/MS, making it an alternative tool for large molecule drug development. With appropriate sample preparation (based on the MW and concentration of the target analyte of interest), immmunocapture LC-MS/MS methods can achieve levels of high sensitivity down to 0.01 to $0.1 \mathrm{nM}$ closer to ELISA assays with a large dynamic range and orthogonal specificity that is generally unaffected by crossreaction issues [61]. In recent comparative studies, Peng et al. developed and validated a simple, sensitive, specific and precise LC-MS/MS assay for quantitation of infliximab PK in human serum by using isotopelabeled signature peptide as the internal standard (IS), which achieved an excellent correlation with ELISA detection [73]. ParK et al. applied LC-MS/MS quadruple time-of-flight mass spectrometric method (QTOF) for the determination of trastuzumab in rat plasma with good sensitivity (LLOD $0.5 \mu \mathrm{g} / \mathrm{ml}$ ) and results in consistence with ELISA assay $\left(R^{2}=0.9104\right)$ [74]. Zhang et al. reported a generic automated LC-MS/MS method for the pharmacokinetic study of a mAb in cynomolgus monkey with comparable data by the immunoassay data as well [75]. Li et al. developed a general LC-MS/MS method approach employing an uniformly heavy-isotope labeled common whole mAb IS and a common immunocapture for sample processing for qualification of four $\operatorname{lgG}(2)$ and four $\lg G(1) \mathrm{mAbs}$. This general LC-MS/MS method approach overcomes the limitations of current methods to reduce time and resources required for preclinical studies [76].

The key benefits of LC-MS/MS over ELISA based method are its high degree of specificity and its ability to simultaneously resolve, detect, and quantify multiple peptides in biological samples or their extracts, as well as even able to identify key metabolites of biologics, in-depth structural characterization and functional insights of therapeutic mAbs, e.g., differentiating biosimilar to originator mAbs as the case of trastuzumab and cetuximab, which will be valuable for biobetters and next generation antibodies design and optimization [77]. Highly selective LC-MS/MS made it possible for simultaneous quantification of several co-administered human antibodies (mAbs), mAb-A and mAb-B of IgG4 subclass in cynomolgus monkey serum with LLOQ around $5-25 \mu \mathrm{g} / \mathrm{mL}$ [78], which cannot be obtained by ELISA assay. As demonstrated in recent examples, $\mathrm{Xu}$ et al. successfully applied a multiplexed hybrid LC-MS/MS pharmacokinetic assay to measure two co-administrated mAbs in a clinical study without requiring 
stringent affinity capture reagents [79]. Lebert and coworkers also demonstrated absolute and multiplexed LC-MS/MS method combining a strategy so-called PSAQ ${ }^{\mathrm{TM}}$ (protein standard absolute quantification or stable isotopically-labeled full-length mAbs) as the ISs for quantification of three mAbs variants with very similar sequences, e.g., IgG1, IgG2 and igG4 isotypes of a lead mAb in rat serum, which can be employed for both preclinical and clinical studies [80]. Moreover, a versatile immunoaffinity LC-MS/MS method was developed to quantify total receptor activator of nuclear factor-kappaB ligand (RANKL) in the presence of denosumab, a humanized monoclonal antibody (mAb) specific to RANKL with an LLOQ of RANKL down to $3.13 \mathrm{ng} / \mathrm{mL}$ for mouse plasma $\mathrm{PK}$, which was not able to determine the total RANKL because the interference of denosumab decreased the recovery of RANKL with commercial ELISA kit [81]. Additionally, 2D-LC (LC $\times$ LC)-MS/MS methodology with improved MS detection limit of approximately two orders of magnitude over direct LC-MS/MS was recommended [82], as well as other LC-MS formats and approaches such as capillary LC-MS gaining MS sensitivity were reviewed [66], offering new opportunities in the analysis of monoclonal antibodies.

On the other hand, mass spectrometry methods (MALDI-TOF, Q-TOF, LTQ-Orbitrap) have been reported for metabolite identification or profiling (MetID) (e.g. deamidation of asparaginyl and glutaminyl residues), preclinical and clinical PK/TK studies with no cross-reaction [61]. Direct analysis of therapeutic monoclonal antibodies, without size reduction through enzyme digestion, is more challenging since these molecules may have a molecular weight up to $150 \mathrm{kDa}$, forming a large distribution of charge states during electrospray ionization (ESI), thus resulting in a complex mass spectrum with decreased sensitivity. To overcome these difficulties, the strategy consists of decreasing their size by proteolytic digestion in order to form 1000 to 2000 Da peptides with few charge states (between 2 and 4) which can be readily quantified in MRM mode on ESI-mass spectrometers. In another recent protocol, detailed mass analysis of structural heterogeneity in monoclonal antibodies at the intact protein level under pseudo-native conditions using native mass spectrometry was reported [83]. This method can be used for different applications such as the analysis of mixtures of mAbs, drug-antibody conjugates and the analysis of mAb PTMs, including the qualitative and quantitative analysis of mAb glycosylation, and it offers several advantages in terms of speed, sensitivity and specificity and high-throughput analysis. Overall, there is a need for general and reliable LC-MS assays capable of supporting the bioanalysis of a variety of human monoclonal antibodybased therapeutics such as reported universal peptide approach to the bioanalysis of human monoclonal antibody protein drug candidates in animal PK/TK studies [84].

\subsection{LC-MS/MS and BLA bioanalysis of ADCS}

Due to the fact that the ADCs are complex mixtures incorporating large and small-molecule characteristics, this poses more unique bioanalytical challenges for $A D C$ than mAb to measure $A D C s$ and their catabolites in plasma and serum [46]. These challenges include ADC bioanalysis such as quantifying $A D C$ and DAR in serum/plasma for PK studies and strategies for assessing immunogenicity. Since ADC species with different DAR distribution may display different potencies, the measured plasma concentrations may not accurately reflect the associated PD effect. As a general rule, both total antibody assay and conjugate antibody assay based on ELISA are needed to measure plasma concentrations over the time. In addition, ADC complexity may increase in in vivo due to biotransformation by catabolism or metabolism, leading to additional changes in DAR or dynamically changing mixtures. Therefore, a standard calibration curve consisting of the reference standard may not be appropriate for quantification of analytes in vivo. Biotransformation may result in $A D C$ analytes in vivo that differ from those in the reference standard in vitro. A notable example was characterization of ADC (trastuzumab emtansine) drug distribution in a cynomolgus monkey PK study by HER2 extracellular domain affinity capture LC-MS, which 
showed the ADR distribution shifts to lower values over time, e.g., DAR=2.76 at day $1,1.86$ at day $10,0.68$ at day 28, respectively [46]. Apparently, the DAR composition of ADC in vivo can vary due to drug deconjugation or metabolic clearance. Therefore, it is important to assess the reference standard calibration curve appropriately for all PK time points to gain accurate DAR and PK data for a better understanding of PD effect. In this case, as suggested by the ADC working group of AAPS and Genentech scientists, a comparative analysis of assay performance for samples prepared with unconjugated antibody and samples prepared with ADC preparations with varying DAR values may be conducted $[46,62]$. Both types of QC samples should ideally produce back-calculated concentrations within the expected acceptable range of the assay (e.g., $\pm 20 \%$ ). In addition, recoveries for QC samples generated using unconjugated antibody and ADC preparations with varying DAR values may be compared [62]. Likewise, Dere et al. demonstrated a comparison of serum T-DM1 concentrations obtained by using the total-trastuzumab and conjugated-trastuzumab ELISA assays using the T-DM1 as the reference standard, which showed an excellent linear regression $\left(R^{2}=0.995\right.$ and a slope $\left.=1.04\right)[60]$.

Often diverse bioanalytical methods, a combination of LBA, high resolution LC-MS and high sensitive LCMS/MS method, are applied to measure ADC related analytes such as drug- antibody conjugate, unconjugated antibody and free toxin as well as metabolite/catabolites. Selecting the appropriate LCMS/MS method of a large molecule largely depends on its molecular features and the required assay sensitivity or expected concentration range in the sample matrix to be analyzed. The affinity capture LC-MS and affinity capture hydrophobic interaction chromatography (HIC) methods developed for ADC characterization provide powerful tools for understanding the fate of ADCs in vivo [85].

\subsection{Matrix interference}

Another bioanalytical challenge is matrix interference with reagents in biological samples in LBA when evaluating PK or ADA, e.g., circulating drug may interfere with the detection of ADA and drug target, or ADA may interfere with quantitation of drug levels in PK/TK bioanalysis. In addition to the most commonly used sample dilution method, the Emergent Technologies Action Program Committee (ETAPC) working group has recently discussed several emerging technologies such as the Singulex ${ }^{\circledR}$ Erenna ${ }^{\circledR}$ platform, Quanterix's proprietary SiMoa' ${ }^{\text {TM }}$ technology (single molecule array), ANP Technologies' NPX4000 Nanoparticles, SQI Diagnostic's Ig PLEX ${ }^{\mathrm{TM}}$ and Genalyte's Maverick ${ }^{\mathrm{TM}}$ technology, the Maverick detection system, to deal with matrix effect on possible false positive or negative ADA detection [86]. Moreover, Zoghbi et al. have developed a breakthrough novel method using precipitation and acid dissociation (PandA) to overcome drug interference in the ADA assay, showing significant improvement over the current approaches in detecting of ADA for two IGg1 and IGg4 drugs as the examples [87]. It was thus claimed that the principle of this novel assay could be used not only for ADA assays but also PK and biomarker (drug target) analysis in the presence of interference factors.

\section{Regulatory considerations}

Regulatory guidance on metabolites and DDI as well as bioanalytical considerations for SMs is well defined [21-23,39,59,88]. In the recent FDA guidance [89], bioanalytical consideration for biopharmaceutics has also been included for industry bioanalytical method validation. General guidelines on preclinical safety evaluations including immunotoxicity of biotechnology-derived pharmaceuticals are available $[34,55,90]$. Currently, it seems no requirement or guidance for metabolic investigation of mAbs from regulatory agencies probably because it is generally accepted that mAbs are unlikely to undergo significant biotransformation. The products of lysosomal degradation of mAbs are assumed to be small peptides, amino acids and small carbohydrates that are readily eliminated by renal excretion or return to the nutrient 
pool without biological effects. However, this assumption may not be true for mAb derivatives such as fusion proteins and antibody-drug conjugates (ADCS) [2]. Indeed, therapeutic protein-drug interactions and implications for drug development have been observed in clinic $[25,91]$. As a consequence, DDI studies on biologics have been outlined in the draft guidance [23], which becomes a major concern for safety evaluations of biologic for investigational new drug (IND) filing. Unlike the mAbs, an additional consideration for $A D C s$ is the $A D M E$ and safety evaluations of novel cytotoxic compounds since the cytotoxic drug or its derivatives can be regarded as the API rather than intermediate during ADC synthesis from regulatory compliance and $C M C$ perspectives. For instance, the unreacted cytotoxic drug or linker might remain in the final $A D C$ drug product. If the concentrations of these impurities are minimal (e.g., $<0.2$ $\%)$, then independent testing of this component may not be necessary [92,93], and vice versa. According to recent suggestions by CDER and AAPS experts [94], free drug related impurities in clinical lot should be qualified relative to data from toxicology studies. Comparable drug/antibody ratios should be maintained between the toxicology lot and the clinical lot. Characterization of the impurity profile of drug/linker intermediates including structure determination of individual impurities (even likely intermediates) at levels $>0.1 \%$ is recommended prior to pivotal clinical trials. Again, given the examples of two approved ADC drugs, e.g. Adcetris and Kadcyla, ADME testing and non-clinical safety assessments of the ADC as well as the cytotoxic drug according to the CMC criteria are essential for IND filing.

Regardless of the regulatory pathway, characterization, comparability, release and stability assays need to be appropriate for the molecule to be analyzed. Before more detailed guidelines on LM bioanalysis are available [89], an alternative LC-MS/MS technique is anticipated to serve as a complementary technique to quantify the antibody or ADC in plasma for more applications of PK determinations as well as the quality control of LMs. Given the fact of increasingly growing interest in biotherapeutics, development and validation of cutting-edge bioanalytical methods are highly desirable for novel mAbs and next generation of ADCs. Strong regulatory guidance and standard industry best practices for ADMET testing of LMs and related bioanalytical considerations are expected to assess and manage the potential risk of biotherapeutics.

\section{Analysis and interpretation of preclinical animal PK of ADCs and human PK prediction}

One ultimate purpose of ADMET testing is to understand the metabolism pathway of target molecule in various in vitro and in vivo species enable to better predict human PK. In general, the human clearance of SMs is more predictable combining in vitro and in vivo animal data by means of well-stirred model and commonly used allometric scaling etc [95-99]. The examples applying allometric scaling have been reviewed for predictions of clearance and volume distribution of therapeutic proteins recently [37]. However, it was argued recently that the utility of allometric scaling and body surface area (BSA) to translate dosage from animal models to human clinical trials are inappropriate for human PK prediction due to interspecies difference in drug metabolism clearance and absorption [100]. For mAbs exhibiting a linear PK without the TMDD, allometric scaling approach applying different scaling exponents is applicable for clearance and volume distribution predictions [101]. Alternatively, PBPK modeling is considered as more accurate human PK predictions for large molecules with the linear PK [102], but it requires a number of input parameters to enable accurate human PK predictions, which limits its applicability in drug development. A recent survey shows that minimal PBPK (mPBPK) model offers a more mechanistic approach using the major structural features of full PBPK models for mAbs in specifically analyzing mAb PK than found in compartment models and provides an intermediary method if a full PBPK model is not available [103]. Comparative evaluations of prediction approaches for projecting human PK of mAbs in early 
drug development prior to the first-in-human (FIH) have been reviewed and decision tree in support of mAb human PK projection has been proposed recently [104]. For LMs with a nonlinear PK involved in the TMDD process, the predictability of plasma and tissue PK can be much more challenging particularly for ADCs. Despite this complexity, a mechanistic framework based on TMDD model was proposed to describe the PK of ADCs and simulate the PK of T-DM1 as the example [105]. Chen et al. [106] have utilized the PBPK modeling as a tool to predict MMAE-based DDI potential drug interactions of ADCs in good agreement with the observed data from clinical DDI data.

In our preliminary analysis of two approved ADCs, it seems no linear regression between $\log (\mathrm{CL})$ and $\log (\mathrm{BW})$ cross species when performing an allometric scaling for either SGN-35 or T-DM1 based on data in Table 2 (further analysis is under way). This may highlights an example of an unpredictable human clearance $(\mathrm{CL})$ from preclinical animal PK data by allometric scaling approach, or at least for these two marked $A D C$ drugs examined herein, due to in different clearance mechanisms of ADCs and SMs. Furthermore, in view of preclinical animal PK data and human PK behaviors of Adcetris (SGN-35) and Kadcyla (T-D1) at two representative doses as summarized in Table 2, it appears that the half-lives of both ADCs in human are relatively shorter than in rodent species such as mouse and rats, but closer to monkey. Also, it should be pointed out that some of reported $t_{1 / 2}$ data are not consistent with the calculated $t_{1 / 2}$ values resulting from a relationship of $V_{d}$ and $C L$, i.e., $\left(t_{1 / 2}=0.693 \times V_{d} / C L\right)$, reflecting the different ways in calculating and reporting $t_{1 / 2}$. Bear in mind that the application of different compartmental models can result in various half-lives, e.g., distribution half-life, elimination or terminal half-life, which should be clarified in PK calculations. Thus, it is important to ensure the reported $t_{1 / 2}$ data in line with the decline of plasma concentration in order to correctly interpret dosing regimen as well as PK/PD relation as addressed above. Moreover, as shown in Table 2 , an increased dose generally results in an increased $t_{1 / 2}$ as a result of a decreased $\mathrm{CL}$ and slightly increased $V_{d}$ in both preclinical species and human. This may be explained by complex clearance mechanisms of biologics by their specific interactions with cellular receptors or described as "TMDD" [11], in addition to possible CYP metabolism resulting from cytotoxin. More specifically, once bound, drugs mainly undergo endocytosis and subsequent lysosomal degradation, dependent on the ratio of receptor/drug concentration, i.e., as the drug level increases or the number of receptors diminishes, the proportion of drug internalized and metabolized decreases, contributing to a lower clearance and consequently longer half-lives. Furthermore, both AUC and Cmax's increment of two ADCs is greater than dose proportional when dose increases in both preclinical species and human. However, no obvious accumulations were observed in TK analysis, which is different from small molecule drugs wherein drug accumulation could often occurs when the CYP metabolism enzymes could be saturated at higher doses by non-linear exposure especially for low clearance compounds.

On the other hand, from PK/PD perspective, it is generally accepted that small drug molecules are rapidly equilibrated at steady state with the same free concentration (unbound) between plasma and tissues, which means that PK/PD can be assessed based on free plasma concentrations without measuring tissue levels. In contrast, therapeutic biologics generally with limited and variable distribution in tissues, and serum levels are not necessarily predictive for tissue levels. Added to that, biologics are administered mainly parenterally, so, ADME testing generally focus on biodistribution or the relationship between tissue concentration and efficacy. As mentioned above, ADCs may have a dose proportional PK of in rats probably due to no specific binding/no cross-reactivity, but a non-linear PK in cynomolgus monkeys due to crossreactivity/specific binding. This has raised a challenge in predicting human PK as well as human dose based on traditional approaches applied for small molecules owing to limited number of species and crossreactivity. In other words, how will preclinical data be translated to clinic in term of human PK and dose 
prediction? In order to better understand the PK/PD relationship and toxicity profile of ADCs, total mAb, $A D C$ or released payload might need to be measured in both plasma and tumor. In addition, conducting PD evaluations requires a clear understanding of the interaction of mAb/ADC with the target at early stage as possible, which may help PK/PD for human efficacy and dose prediction. PK/PD modeling and simulation (M\&S) may provide an excellent tool to overcome these challenges, as it can simultaneously integrate the PK/PD of ADCs and their components in a quantitative manner [111,112]. Additionally, the computational PK/PD models can also serve as a cornerstone for the model-based drug development and preclinical-toclinical translation of ADCs. For instance, Shah et al. has developed a mechanistic model able to predict clinical response by integrating all preclinical biomeasures and PK/PD data of Brentuximab-vedotin [113]. Khot et al. highlighted the applications of M\&S to gain distinct insights into ADC development for PK/PD and toxicodynamic data by integrating a diverse array of in vitro, preclinical and clinical data generated at different stages of ADC development [114].

Table 2. Preclinical and human PK data of ADC drugs (Adcetris and Kadcyla)*

\begin{tabular}{|c|c|c|c|c|c|c|c|c|c|}
\hline Drug & & Mc & & & & Mo & rey & & Ian \\
\hline \multirow{7}{*}{$\begin{array}{l}\text { Adcetris } \\
\text { (brentuximab } \\
\text { vedotin, SGN-35 ) }\end{array}$} & Dose $\left(\mathrm{mg} \mathrm{kg}^{-1}\right)$ & 10 & - & 0.5 & 5.0 & 0.3 & 1.0 & 1.2 & 2.7 \\
\hline & $C_{\max }\left(\mu g \mathrm{~mL}^{-1}\right)$ & - & - & 13 & 171 & 6.9 & 29.2 & 18.9 & 45.7 \\
\hline & $\mathrm{A} \cup C\left(\mu \mathrm{g} \mathrm{mL} L^{-1}\right.$ day $\left.^{-1}\right)$ & 2313 & - & 20 & 253 & 11 & 55.3 & 46.1 & 125.8 \\
\hline & $t_{1 / 2}$ (days) & 16.9 & - & 14.6 & 8.5 & 1.8 & 2.7 & 3.8 & 6.0 \\
\hline & $t_{1 / 2}($ calculated $)$ & 16.9 & - & 5.1 & 4.7 & 1.7 & 2.5 & 3.8 & 6.0 \\
\hline & $\mathrm{CL}\left(\mathrm{mL}\right.$ day $\left.^{-1} \mathrm{~kg}^{-1}\right)$ & 4.4 & - & 25 & 20 & 27.7 & 18.5 & 26.0 & 21.5 \\
\hline & $V_{s s}\left(\mathrm{~mL} \mathrm{~kg}^{-1}\right)$ & 107 & - & 183 & 135 & 68 & 67.4 & 142 & 186 \\
\hline \multirow{7}{*}{$\begin{array}{l}\text { Kadcyla (ado- } \\
\text { trastuzumab } \\
\text { emtansine, T- }\end{array}$} & Dose $\left(\mathrm{mg} \mathrm{kg}^{-1}\right)$ & 0.3 & 15 & 0.3 & 20 & 0.3 & 3.0 & 0.3 & 4.8 \\
\hline & $C_{\max }\left(\mu g \mathrm{~mL}^{-1}\right)$ & - & - & - & - & 7.4 & 74.2 & 9.63 & 130 \\
\hline & $\mathrm{AUC}\left(\mu \mathrm{g} \mathrm{mL}^{-1} \mathrm{day}^{-1}\right)$ & - & - & - & - & 7.5 & 180 & 14.5 & 673 \\
\hline & $t_{1 / 2}$ (days) & 4.2 & 13.1 & 4.9 & 5.4 & 0.9 & 2.9 & 1.3 & 4.1 \\
\hline & $t_{1 / 2}$ (calculated) & 2.2 & 2.3 & 5.0 & 4.7 & 0.8 & 2.5 & 1.2 & 4.0 \\
\hline & $\mathrm{CL}\left(\mathrm{mL} \mathrm{day}^{-1} \mathrm{~kg}^{-1}\right)$ & 13.0 & 19.2 & 10.1 & 22.1 & 40.4 & 16.5 & 21.1 & 7.1 \\
\hline & $V_{\mathrm{ss}}\left(\mathrm{mL} \mathrm{kg}{ }^{-1}\right)$ & 40.5 & 62.9 & 72.9 & 149 & 44.2 & 60.7 & 35.7 & 41.2 \\
\hline
\end{tabular}

*Adcetris/SGN-35 mouse PK data from ref. [47], rat and monkey PK data from ref. [107] and human PK data from ref. [108]. Kadcyla/TDM1 mouse and rat PK data from ref. [109], monkey PK data from ref. [110] and human PK data from ref. [58]. $t_{1 / 2}$ (calculated) data obtained by the relationship between $V_{\mathrm{d}}$ and $\mathrm{CL}$ data $\left(t_{1 / 2}=0.693 V_{\mathrm{d}} / \mathrm{CL}\right)$.

Despite considerable differences between preclinical PK data and unpredictable human PK as well as complex PK/PD implication, Saber and Leighton recently reported an FDA analysis of ADCs on FIH dose selection [115]. Based on an FDA oncology analysis of INDs for ADCs using preclinical data (plasma stability, toxicities in animals, and toxicology study designs), it was concluded that ADCs can share the same small molecule drug concerning FIH dose selection. More specifically, selecting a FIH dose such as $1 / 6$ th the 
HNSTD in cynomolgus monkeys or 1/10th the STD10 in rodents scaled according to BSA generally resulted in the acceptable balance of safety and efficient dose-escalation in FIH trial. This certainly provides an encouraging guideline to support the safety evaluation of ADCs and FIH dose selection for clinical development by similar approach as small molecule drugs in this aspect.

\section{Concluding remarks}

Biologic large molecules hold unique characteristics from small chemical entities. Overall, the principles of ADME testing for SMs can be applied to testing biologics such as mAbs and ADCs. However, there are remarkable differences between SMs and LMs in ADME assays associated with bioanalytical strategies. In comparison with ADME testing of SMs, there are limited in vitro models for ADME testing of LMs. Also, fewer species with cross-reactivity can be utilized for in vivo PK studies as well as safety evaluations for LMs. For initial ADC evaluation, In vitro plasma stability assay combined with monitoring the free toxin released from $A D C$ in in vivo plasma can be utilized as a surrogate before conducting complicated and labintensive bioanalytical method development for PK quantification. From ADME perspective, the design of novel linkers and the application of new conjugation technologies for uniform DAR can not only further improve $A D C$ stability and consequent PK profiles as well as tolerability in systemic circulation of ADCs, but also make bioanalysis of ADC relatively easier.

General ADME/PK testing and nonclinical toxicity studies should be conducted for both $A D C$ and cytotoxin as appropriate, as exemplified in two approved ADC drugs (Adcetris and Kadcyla). Owing to fact that many elements such as antibody, free toxin, linker stability as well as product attributes can be ADC toxicity determinants, ADME testing and safety evaluations of ADC are much more complex with significant development challenges, requiring unique and customized nonclinical ADME approaches that differs from classical ADME studies for SMs. It appears that safety evaluation of key drug metabolite for ADC drugs may be considered as a minor issue as long as the released cytotoxic concentration is far below its MTD concentration. However, the DDI studies on mAbs or ADCs (both ADC and cytotoxic drug) are strongly recommended by FDA guidance because of increased use of biotherapeutics and clear evidence of proteindrug interactions observed in clinic. Relevant tools need to be developed for early DDI assessment of mAbs or ADCs with small molecules and other biologics for predicting clinical risk. Additionally, it is anticipated that new methods are likely to be developed for a much better understanding of the immunotoxic potential from preclinical observations to clinic relevance.

PK of LMs is often PD-dependent and it might have a dose proportional PK owing to no specific binding/no cross-reactivity or otherwise non-linear PK due to specific binding/cross-reactivity. Currently, the prediction of human PK and translation of preclinical PK/PD models to clinic are challenging especially for ADCs due to limited species with cross-reactivity as well as complex TMDD and CYP enzymes based metabolism pathways. In addition, it requires simultaneous quantitative understanding about the PK/PD properties of three different molecular species, i.e., the monoclonal antibody, the drug, and the conjugate in both serum and tissues. Further understanding PK prediction models of LMs and refining PK/PD based on M\&S are needed for human PK and efficacy predictions and dose optimization.

Currently, LBA-based ELISA is primarily preferred method for bioanalysis of mAbs on available highthroughput formats. Whilst LC-MS/MS can be utilized as an alternative technology of unique advantages for simulations quantification of co-administrated mAbs or in the case of low recovery observed due to matrix interference with ELISA method. Although the routine usage of LC-MS is mainly hampered by the relatively time-consuming method development due to complex sample preparations, the availability of isotopically labeled proteins as ISs, immunocapture and enzyme digestion of LMs as well as limited sensitivity as 
compared to a typical BLA, the highly selective LC-MS has been emerging as a viable alternative for qualitative and quantitative applications of LMs, which can achieve comparable detection sensitivity with ELISA for PK quantification of mAbs as demonstrated in a number of recent applications. Several multiple approaches have proposed to mitigate interference issues in LBA, including the use of LC-MS for bioanalysis of mAbs. However, it is desirable to deploy only one assay, rather than multiple assays, especially in later stages of clinical development. Due to the heterogeneous nature of ADCs and potential biotransformation, the hybrid technologies of best LBA and LC-MS/MS are imperative for molecular characterization, PK/TK bioanalysis including total antibody, total $A D C$, antibody-conjugated payload and unconjugated payload and immunogenicity evaluation as well as biotransformation and safety assessments of ADCs.

\section{Acknowledgements}

Author would like to thank his colleagues Xiaoyan Zhu, Zhendong Xue, Jun Feng, Kaijie He and Yuchang Mao for helping with some references, especially Dr. Weikang Tao for his valuable comments and critical review of this manuscript, and Dr. Kin Tam for final proof-reading of this manuscript.

\section{Declaration of interest}

This article solely reflects author's personal opinions. The author declares no competing conflicts of interest and receives no financial support from any affiliations in preparing this manuscript.

\section{References}

[1] A. Beck, J.M. Reichert. MAbs 6 (2014) 15-17.

[2] J.W. Lee. Bioanalysis 5 (2013) 2003-2014.

[3] H.L. Perez, P.M. Cardarelli, S. Deshpande, S. Gangwar, G.M. Schroeder, G.D. Vite, R.M. Borzilleri. Drug Discov. Today 19 (2014) 869-881.

[4] J.M. Reichert. MAbs 7 (2015) 1-8.

[5] T. Prueksaritanont, C. Tang. AAPS J 14 (2012) 410-419.

[6] K. Thudium, S. Bilić, D. Leipold, W. Mallet, S. Kaur, B. Meibohm, H. Erickson, J. Tibbitts, H. Zhao, M. Gupta. MAbs 5 (2013) 5-12.

[7] A. Mullard. Nat. Rev. Drug Discov. 12 (2013) 329-332.

[8] J.A. Cavagnaro. Nat. Rev. Drug Discov. 1 (2002) 469-475.

[9] T.H. Han, B. Zhao. Drug Metab. Dispos. 42 (2014) 1914-1920.

[10] S. Shi. Curr. Drug Metab. 15 (2014) 271-290.

[11] E. Ezan, F. Becher, F. Fenaille. Expert Opin. Drug Metab. Toxicol. 10 (2014) 1079-1091.

[12] H. Wan. ADMET \& DMPK 1 (2013) 19-28.

[13] T. Tuntland, B. Ethell, T. Kosaka, F. Blasco, R.X. Zang, M. Jain, T. Gould, K. Hoffmaster. Front. Pharmacol. 5 (2014) 174.

[14] B. Feng, M.V. Varma, C. Costales, H. Zhang, L. Tremaine. Expert Opin. Drug Discov. 9 (2014) 873-890.

[15] V. Ahuja, S. Sharma. J. Appl. Toxicol. 34 (2014) 576-594.

[16] M. Meng, L. Wang, T. Voelker, S. Reuschel, K.C. Van Horne, P. Bennett. Bioanalysis 5 (2013) 91-115.

[17] L. Li, D. Tian, F. Chen, J. Yang, K. Yu, Y. Sun. Curr. Drug Metab. 13 (2012) 1206-1212.

[18] H. Wan, A.G. Holmen. Comb. Chem. High Throughput Screen. 12 (2009) 315-329.

[19] H. Wan, M.J. Rehngren. J. Chromatogr. A 1102 (2006) 125-134.

[20] H. Wan, M. Rehngren, F. Giordanetto, F. Bergström, A. Tunek. J. Med. Chem. 50 (2007) 4606-4615. 
[21] FDA Guidance for Industry: Safety testing of drug metabolites. (2008) Available from: http:// www.fda.gov/downloads/drugs/guidancecomplianceregulatoryinformation/guidances/ucm079266.p df (accessed on March 23, 2016).

[22] ICH Guidance on nonclinical safety studies for the conduct of human clinical trials and marketing authorization for pharmaceuticals. (2010) Available from:

http://www.fda.gov/downloads/drugs/guidancecomplianceregulatoryinformation/guidances/ucm07 3246.pdf (accessed on March 23, 2016).

[23] FDA Guidance for Industry: Drug Interaction Studies - Study Design, Data Analysis, Implications for dosing, and Labeling Recommendation. (2012) Available from:

http://www.fda.gov/downloads/drugs/guidancecomplianceregulatoryinformation/guidances/ucm29 2362.pdf (accessed on March 23, 2016).

[24] K. Lin, J. Tibbitts. Pharm. Res. 29 (2012) 2354-2366.

[25] S.M. Huang, H. Zhao, J.I. Lee, K. Reynolds, L. Zhang, R. Temple, L.J. Lesko. Clin. Pharmacol. Ther. 87 (2010) 497-503.

[26] I.A. Kaltashov, C.E. Bobst, R.R. Abzalimov, G. Wang, B. Baykal, S. Wang. Biotechnol. Adv. 30 (2012) 210-222.

[27] D. Nebija, H. Kopelent-Frank, E. Urban, C.R. Noe, B. Lachmann. J. Pharm. Biomed. Anal. 56 (2011) 684-691.

[28] J. Visser, I. Feuerstein, T. Stangler, T. Schmiederer, C. Fritsch, M. Schiestl. BioDrugs 27 (2013) 495507.

[29] M.M. Sun, K.S. Beam, C.G. Cerveny, K.J. Hamblett, R.S. Blackmore, M.Y. Torgov, F.G.M. Handley, N.C. Ihle, P.D. Senter, S.C. Alley. Bioconjug. Chem. 16 (2005) 1282-1290.

[30] R.J. Harris, B. Kabakoff, F.D. Macchi, F.J. Shen, M. Kwong, J.D. Andya, S.J. Shire, N. Bjork, K. Totpal, A.B. Chen. J. Chromatogr. B Biomed. Sci. Appl. 752 (2001) 233-245.

[31] J. Vlasak, R. lonescu. Curr. Pharm. Biotechnol. 9 (2008) 468-481.

[32] S.R. Trevino, J.M. Scholtz, C.N. Pace. J. Pharm. Sci. 97 (2008) 4155-4166.

[33] T.J. Gibson, K. McCarty, I.J. McFadyen, E. Cash, P. Dalmonte, K.D. Hinds, A.A. Dinerman, J.C. Alvarez, D.B. Volkin. J. Pharm. Sci. 100 (2011) 1009-1021.

[34] FDA. Guidance for Industry: Assay Development for Immunogenicity Testing of Therapeutic Proteins. (2009) Available from:

http://www.fda.gov/downloads/drugs/guidancecomplianceregulatoryinformation/guidances/ucm19 2750.pdf. (accessed on March 23, 2016).

[35] FDA. Guidance for Industry: Immunotoxicology evaluation of investigation new drug. (2002) Available from:

http://www.fda.gov/downloads/drugs/guidancecomplianceregulatoryinformation/guidances/ucm07 9239.pdf. (accessed on March 23, 2016).

[36] E.D. Lobo, R.J. Hansen, J.P. Balthasar. J. Pharm. Sci. 93 (2004) 2645-2668.

[37] J.H. Lin. Curr. Drug Metab. 10 (2009) 661-691.

[38] Y. Vugmeyster, X. Xu, F.P. Theil, L.A. Khawli, M.W. Leach. World J. Biol. Chem. 3 (2012) 73-92.

[39] R. Deng, F. Jin, S. Prabhu, S. Iyer. Expert Opin. Drug Metab. Toxicol. 8 (2012) 141-160.

[40] J.D. Gomez-Mantilla, I.F. Troconiz, Z. Parra-Guillen, M.J. Garrido. J. Pharmacokinet. Pharmacodyn. 41 (2014) 523-536.

[41] S.A. Roberts, P.A. Andrews, D. Blanset D, K.M. Flagella, B. Gorovits, C.M. Lynch, P.L. Martin, K. Kramer-Stickland, S. Thibault, G. Warner. Regul. Toxicol. Pharmacol. 67 (2013) 382-391.

[42] P. Chamberlain. Bioanalysis 5 (2013) 561-574.

[43] C.A. Boswell, E.E. Mundo, C. Zhang, D. Bumbaca, N.R. Valle, K.R. Kozak, A. Fourie, J. Chuh, N. Koppada, O. Saad, H. Gill, B.Q. Shen, B. Rubinfeld, J. Tibbitts, S. Kaur, F.P. Theil, P.J. Fielder, L.A. Khawli, K. Lin. Bioconjug. Chem. 22 (2011) 1994-2004. 
[44] E. Chertova, C. Bergamaschi, O. Chertov, R. Sowder, J. Bear, J.D. Roser, R.K. Beach, J.D. Lifson, B.K. Felber, G.N. Pavlakis. J. Biol. Chem. 288 (2013) 18093-18103.

[45] J. Gaudreault, D. Fei, J. Rusit, P. Suboc, V. Shiu. Invest. Ophthalmol. Vis. Sci. 46 (2005) 726-733.

[46] S. Kaur, K. Xu, O.M. Saad, R.C. Dere, M. Carrasco-Triguero. Bioanalysis 5 (2013) 201-226.

[47] K.J. Hamblett, P.D. Senter, D.F. Chace, M.M.C. Sun, J. Lenox, C.G. Cerveny, K.M. Kissler, S.X. Bernhardt, A.K. Kopcha, R.F. Zabinski, D.L. Meyer, J.A. Francisco. Clin. Cancer Res. 10 (2004) 70637070.

[48] M. Dorywalska, P. Strop, J.A. Melton-Witt, A. Hasa-Moreno, S.E. Farias, M.G. Casas, K. Delaria, V. Lui, K. Poulsen, C. Loo, S. Krimm, G. Bolton, L. Moine, R. Dushin, T.T. Tran, S.H. Liu, M. Rickert, D. Foletti, D.L. Shelton, J. Pons, A. Rajpal. Bioconjug. Chem. 26 (2015) 650-659.

[49] A.G. Polson, J. Calemine-Fenaux, P. Chan, W. Chang, E. Christensen, S. Clark, F.J. de Sauvage, D. Eaton, K. Elkins, J.M. Elliott, G. Frantz, R.N. Fuji, A. Gray, K. Harden, G.S. Ingle, N.M. Kljavin, H. Koeppen, C. Nelson, S. Prabhu, H. Raab, S. Ross, J.P. Stephan, S.J. Scales, S.D. Spencer, R. Vandlen, B. Wranik, S.F. Yu, B. Zheng, A. Ebens. Cancer Res. 69 (2009) 2358-2364.

[50] J.R. Junutula, H. Raab, S. Clark, S. Bhakta, D.D. Leipold, S. Weir, Y. Chen, M. Simpson, S.P. Tsai, M.S. Dennis, Y. Lu, Y.G. Meng, C. Ng, J. Yang, C.C. Lee, E. Duenas, J. Gorrell, V. Katta, A. Kim, K. McDorman, K. Flagella, R. Venook, S. Ross, S.D. Spencer, W.L. Wong, H.B. Lowman, R. Vandlen, M.X. Sliwkowski, R.H. Scheller, P. Polakis, W. Mallet. Nat. Biotechnol. 26 (2008) 925-932.

[51] N.K. Damle. Nat. Biotechnol. 26 (2008) 884-885.

[52] P. Dennler, A. Chiotellis, E. Fischer, D. Brégeon, C. Belmant, L. Gauthier, F. Lhospice, F. Romagne, R. Schibli. Bioconjug. Chem. 25 (2014) 569-578.

[53] P.F. Peddi, S.A. Hurvitz. Future Oncol. 9 (2013) 319-326.

[54] H.K. Erickson, J.M. Lambert. AAPS J. 14 (2012) 799-805.

[55] ICH. Guidance for Industry: S6(R1): preclinical safety evaluation of biotechnology-derived pharmaceuticals. (2012) Available from:

http://www.fda.gov/downloads/Drugs/GuidanceComplianceRegulatoryInformation/Guidances/UCM 194490.pdf. (accessed on March 23, 2016).

[56] M.B. Hock, K.E. Thudium, M. Carrasco-Triguero, N.F. Schwabe. AAPS J. 17 (2015) 35-43.

[57] FDA. Guidance for Industry: S9 Nonclinical Evaluation for Anticancer Pharmaceuticals. (2010)

Available from:

http://www.fda.gov/downloads/drugs/guidancecomplianceregulatoryinformation/guidances/ucm08 5389.pdf. (accessed on March 23, 2016).

[58] S. Girish, M. Gupta, B. Wang, D. Lu, I.E. Krop, C.L. Vogel, H.A. Burris III, P.M. LoRusso, J.H. Yi, O. Saad, B. Tong, Y.W. Chu, S. Holden, A. Joshi. Cancer Chemother. Pharmacol. 69 (2012) 1229-1240.

[59] FDA. Guidance for Industry: Bioanalytical Method Validation. (2001) Available from:

http://www.fda.gov/downloads/drugs/guidancecomplianceregulatoryinformation/guidances/ucm0701 07.pdf. (accessed on March 23, 2016).

[60] R. Dere, J.H. Yi, C. Lei, O.M. Saad, C. Huang, Y. Li, J. Baudys, S. Kaur. Bioanalysis 5 (2013) 1025-1040.

[61] E. Ezan, M. Dubois, F. Becher. Analyst 134 (2009) 825-834.

[62] B. Gorovits, S.C. Alley, S. Bilić, B. Booth, S. Kaur, P. Oldfield, S. Purushothama, C. Rao, S. Shord, P. Siguenza. Bioanalysis 5 (2013) 997-1006.

[63] Y.M. Wang, V. Jawa, M. Ma. Bioanalysis 6 (2014) 79-87.

[64] L. Stevenson, L. Amaravadi, H. Myler, L. Salazar-Fontana, B. Gorovits, S. Kirshner, L. Xue, F. Garofolo, S.C. Alley, T. Thway, A. Joyce, S. Bansal, C. Beaver, A. Bergeron, X.Y. Cai, L. Cojocaru, B. DeSilva, I.Dumont, E. Fluhler, S. Fraser, D. Gouty, S. Gupta, S. Haidar, R. Hayes, B. Ingelse, A. Ishii-Watabe, S. Kaur, L. King, O. Laterza, S. Leung, A. Lévesque, M. Ma, C. Petit-Frere, R. Pillutla, M. Rose, G. Schultz, J. Smeraglia, S. Swanson, A. Torri, F. Vazvaei, J. Wakelin-Smith, A. Wilson, E. Woolf, T.Y. Yang. Bioanalysis 6 (2014) 3355-3368. 
[65] L. Stevenson, F. Garofolo, B. DeSilva, I. Dumont, S. Martinez, M. Rocci, L. Amaravadi, M. BrudnyKloeppel, A. Musuku, B. Booth, C. Dicaire, L. Wright, L. Mayrand-Provencher, M. Losauro, D. Gouty, M. Arnold, S. Bansal, S. Dudal, D. Dufield, J. Duggan, C. Evans, E. Fluhler, S. Fraser, B. Gorovits, S. Haidar, R. Hayes, S. Ho, R. Houghton, R. Islam, R. Jenkins, N. Katori, S. Kaur, M. Kelley, M. Knutsson, J. Lee, H. Liu, S. Lowes, M. Ma, A. Mikulskis, H. Myler, B. Nicholson, T. Olah, E. Ormsby, S. Patel, V. Pucci, C. Ray, G. Schultz, J. Shih, R. Shoup, C. Simon, A. Song, J.T. Neto, V. Theobald, T. Thway, J. Wakelin-Smith, J. Wang, L. Wang, J. Welink, E. Whale, E. Woolf, R. Xu. Bioanalysis 5 (2013) 29032918.

[66] D. L. Chappell, M.E. Lassman, T. McAvoy, M. Lin, D.S. Spellman, O.F. Laterza. Bioanalysis 6 (2014) 1843-1857.

[67] Y.Q. Xiao. MOJ Immunol. 1 (2014) 4.

[68] Quantitation of Antibody-Drug Conjugates, CYROS Application Note, D0020478.

[69] A.P. Joyce, M. Wang, R. Lawrence-Henderson R, C. Filliettaz, S.S. Leung, X. Xu, D.M. O'Hara. Pharm. Res. 31 (2014) 1823-1833.

[70] J. H. Heo, X. Mou, F. Wang, J.M. Troisi, C.W. Sandifer, S. Kirby, D. Driscoll, S. Mercorelli, D.J. Pollard. Pharm. Bioprocess. 2 (2014) 129-139.

[71] C.M. Hall, J.T. Pearson, V. Patel, L.C. Wienkers, R.J. Greene. J. Immunol. Methods 393 (2013) 70-73.

[72] R. Piparia, D. Ouellette, W.B. Stine, C. Grinnell, E. Tarcsa, C. Radziejewski, I. Correia. MAbs 4 (2012) 521-531.

[73] X. Peng, B. Liu, Y. Li, H. Wang, X. Chen, H. Guo, Q. Guo, J. Xu, H. Wang, D. Zhang, J. Dai, S. Hou, Y. Guo. Chromatographia 78 (2015) 521-531.

[74] M.H. Park, M.W. Lee, Y.G. Shin. Biomed. Chromatogr. 2015.

[75] Q. Zhang, D.S. Spellman, Y. Song, B. Choi, N.G. Hatcher, D. Tomazela, M. Beaumont, M. Tabrizifard, D. Prabhavalkar, W. Seghezzi, J. Harrelson, K.P. Bateman. Anal. Chem. 86 (2014) 8776-8784.

[76] H. Li, R. Ortiz, L. Tran, M. Hall, C. Spahr, K. Walker, J. Laudemann, S. Miller, H. Salimi-Moosavi, J.W. Lee. Anal. Chem. 84 (2012) 1267-1273.

[77] A. Beck, S. Sanglier-Cianferani, A. Van Dorsselaer. Anal. Chem. 84 (2012) 4637-4646.

[78] H. Jiang, J. Zeng, C. Titsch, K. Voronin, B. Akinsanya, L. Luo, H. Shen, D.D. Desai, A. Allentoff, A.F. Aubry, B.S. DeSilva, M.E. Arnold. Anal. Chem. 85 (2013) 9859-9867.

[79] K. Xu, L. Liu, M. Maia, J. Li, J. Lowe, A. Song, S. Kaur. Bioanalysis 6 (2014) 1781-1794.

[80] D. Lebert, G. Picard, C, Beau-Larvor, L. Troncy, C. Lacheny, B. Maynadier, W. Low, N. Mouz, V. Brun, C. Klinguer-Hamour, M. Jaquinod, A. Beck. Bioanalysis 7 (2015) 1237-1251.

[81] I. Onami, M. Ayabe, N. Murao, M. Ishigai. J. Chromatogr. A 1334 (2014) 64-71.

[82] K. Sandra, P. Sandra. Bioanalysis 7 (2015) 2843-2847.

[83] S. Rosati, Y. Yang, A. Barendregt, A.J.R. Heck. Nat. Protoc. 9 (2014) 967-976.

[84] M.T. Furlong, S. Zhao, W. Mylott, R. Jenkins, M. Gao, V. Hegde, J. Tamura, A. Tymiak, M. Jemal. Bioanalysis 5 (2013) 1363-1376.

[85] A. Liu, A. Kozhich, D. Passmore, H. Gu, R. Wong, F. Zambito, V.S. Rangan, H. Myler, A.F. Aubry, M.E. Arnold, J. Wang. J. Chromatogr. B Analyt. Technol. Biomed. Life Sci. 1002 (2015) 54-62.

[86] R.M. Crisino, L. Luo, B. Geist, J. Zoghbi, F. Spriggs. Bioanalysis 6 (2014) 1033-1036.

[87] J. Zoghbi, Y. Xu, R. Grabert, V. Theobald, S. Richards. J. Immunol. Methods 426 (2015) 62-69.

[88] FDA. Guidance for Industry: Nonclinical Studies for the Safety Evaluation of Pharmaceutical Excipients. (2005) Available from:

http://www.fda.gov/downloads/drugs/guidancecomplianceregulatoryinformation/guidances/ucm07 9250.pdf. (accessed on March 23, 2016).

[89] FDA. Guidance for Industry: Bioanalytical Method Validation, Biopharmaceutics. Revision 1. (2013) Available from:

http://www.fda.gov/downloads/Drugs/GuidanceComplianceRegulatoryInformation/Guidances/UCM 368107.pdf. (accessed on March 23, 2016). 
[90] K.L. Hastings, Int. Immunopharmacol. 2 (2002) 1613-1618.

[91] K. Seitz, H. Zhou. J. Clin. Pharmacol. 47 (2007) 1104-1118.

[92] ICH. Guidance for Industry: Q3B(R2) Impurities in New Drug Products.2006. Available from: http://www.fda.gov/downloads/drugs/guidancecomplianceregulatoryinformation/guidances/ucm07 3389.pdf. (accessed on March 23, 2016).

[93] ICH. Guidance for Industry: Q3A Impurities in New Drug Substances.2008. Available from: http://www.fda.gov/downloads/drugs/guidancecomplianceregulatoryinformation/guidances/ucm07 3385.pdf. (accessed on March 23, 2016).

[94] Regulatory Considerations for Antibody-Drug Conjugates, OPS, CDER, AAPS (October 18, 2012). Available from:

http://www.fda.gov/downloads/aboutfda/centersoffices/officeofmedicalproductsandtobacco/cder/ ucm341177.pdf. (accessed on March 23, 2016).

[95] I. Mahmood. Clin. Pharmacokinet. 51 (2012) 527-542.

[96] H. Wan, P. Bold, L.O. Larsson, J. Ulander, S. Peters, B. Lofberg, A.L. Ungell, M. Nagard, A. Llinas. Curr. Drug Metab. 11 (2010) 583-594.

[97] V.K. Sinha, S.S. De Buck, L.A. Fenu, J.W. Smit, M. Nijsen, R.A.H.J. Gilissen, A. Van Peer, K. Lavrijsen, C.E. Mackie. Clin. Pharmacokinet. 47 (2008) 35-45.

[98] Q. Huang, J.E. Riviere. Expert Opin. Drug Metab. Toxicol. 10 (2014) 1241-1253.

[99] L. Liu, K.S. Pang. Eur. J. Pharm. Sci. 29 (2006) 215-230.

[100] O.L. Blanchard, J.M. Smoliga. FASEB J. 29 (2015) 1629-1634.

[101] L. Diao, B. Meibohm. Expert Opin. Drug Metab. Toxicol. 11 (2015) 1115-1125.

[102] H.M. Jones, Y. Chen, C. Gibson, T. Heimbach, N. Parrott, S.A. Peters, J. Snoeys, V.V. Upreti, M. Zheng S.D. Hall. Clin. Pharmacol. Ther. 97 (2015) 247-262.

[103] Y. Cao, W.J. Jusko. J. Pharmacokinet. Pharmacodyn. 41 (2014) 571-580.

[104] J. Wang, S. Iyer, P.J. Fielder, J.D. Davis, R. Deng. Biopharm. Drug Dispos. 2015.

[105] L. Gibiansky, E. Gibiansky. J. Pharmacokinet. Pharmacodyn. 41 (2014) 35-47.

[106] Y. Chen, D. Samineni, S. Mukadam, H. Wong, B.Q. Shen, D. Lu, S. Girish, C. Hop, J.Y. Jin, C. Li. Clin. Pharmacokinet. 54 (2015) 81-93.

[107] ADCETRIS, FDA CDER (CENTER FOR DRUG EVALUATION AND RESEARCH), PHARMACOLOGY REVIEW, application number 1253990rig1s000, 2011. Available from: http://www.accessdata.fda.gov/drugsatfda_docs/label/2011/125388s000,125399s000lbl.pdf.

[108] ADCETRIS, FDA CDER (CENTER FOR DRUG EVALUATION AND RESEARCH), CLINICAL PHARMACOLOGY and BIOPHARMACEUTICS REVIEW, application number 1253990rig1s000, 2011. Available from:http://www.accessdata.fda.gov/drugsatfda_docs/label/2011/125388s000,125399s000lbl.pdf. (accessed on March 23, 2016).

[109] Kacyla EMA-Assessment report on Kadcyla-EMA749228-2013. Available from: http://www.ema.europa.eu/docs/en_GB/document_library/EPAR__Public_assessment_report/human/002389/WC500158595.pdf. (accessed on March 23, 2016).

[110] KADCYLA, FDA CDER (CENTER FOR DRUG EVALUATION AND RESEARCH), PHARMACOLOGY REVIEW, application number 1254270rig1s000, 2013. Available from: http://www.accessdata.fda.gov/drugsatfda_docs/label/2013/125427lbl.pdf. (accessed on March 23, 2016).

[111] A.P. Singh, Y.G. Shin, D.K. Shah. Pharm. Res. 32 (2015) 3508-3525 .

[112] P. Sapra, A. Betts, J. Boni. Expert Rev. Clin. Pharmacol. 6 (2013) 541-555.

[113] D.K. Shah, N. Haddish-Berhane, A. Betts. J. Pharmacokinet. Pharmacodyn. 39 (2012) 643-659.

[114] A. Khot, S. Sharma, D.K. Shah. Bioanalysis 7 (2015) 1633-1648.

[115] H. Saber, J.K. Leighton. Regul. Toxicol. Pharmacol. 71 (2015) 444-452. 


\begin{tabular}{|c|c|}
\hline AAPS & American Association of Pharmaceutical Scientists \\
\hline ADA & anti-drug antibody \\
\hline ADC & Antibody-drug conjugates \\
\hline ADME & Absorption, distribution, metabolism and elimination \\
\hline API & Active pharmaceutical ingredient \\
\hline ATA & Anti-therapeutic antibodies \\
\hline AUC & Area under the curve \\
\hline BSA & Body surface area \\
\hline CDER & Center for drug evaluation and research \\
\hline CYP & Cytochrome P450 \\
\hline DAR & Drug-antibody ratio, payload \\
\hline DDI & Drug-drug interaction \\
\hline ETAPC & The Emergent Technologies Action Program Committee \\
\hline ELISA & Enzyme-linked immunesorbent assay \\
\hline FIH & First-in-human \\
\hline hERG & Human ether-a-go-go related gene \\
\hline HIC & Hydrophobic interaction chromatography \\
\hline HNSTD & Highest non-severely toxic dose \\
\hline LBA & Ligand binding assay \\
\hline IgG & Immunoglobulin G \\
\hline IM & Intramuscular \\
\hline IND & Investigational new drug \\
\hline IS & Internal standard \\
\hline IV & Intravenous \\
\hline LC-MS & Liquid chromatography-mass spectrometry \\
\hline LC-MS/MS & Liquid chromatography with tandem mass spectrometry \\
\hline LMs & Large molecules \\
\hline mAb & Monoclonal antibody \\
\hline MMAE & Monomethyl auristatin E \\
\hline M\&S & Modeling and simulation \\
\hline MTD & Maximum tolerated dose \\
\hline MW & Molecular weight \\
\hline PBPK & Physiologically-based pharmacokinetics \\
\hline PCC & Preclinical candidate \\
\hline PD & Pharmacodynamics \\
\hline PK & Pharmacokinetics \\
\hline PK/PD & Pharmacokinetics-pharmacodynamics \\
\hline PK/TK & Pharmacokinetics-toxicokinetics \\
\hline PTM & Post-translational modifications \\
\hline $\mathrm{SC}$ & Subcutaneous \\
\hline SMs & Small molecules \\
\hline TDC & ThioMab-drug conjugates \\
\hline T-DM1 & Trastuzumab-emtansine \\
\hline TMB & 3, 3', 5, 5' - Tetramethylbenzidine \\
\hline TMDD & Target-mediated drug disposition \\
\hline$V_{d}$ & Volume of distribution \\
\hline
\end{tabular}

(C)2016 by the authors; licensee IAPC, Zagreb, Croatia. This article is an open-access article distributed under the terms and conditions of the Creative Commons Attribution license (http://creativecommons.org/licenses/by/3.0/) (cc) E EY 